\title{
Regularity of center-of-pressure trajectories depends on the amount of attention invested in postural control
}

\author{
Stella F. Donker · Melvyn Roerdink • \\ An J. Greven · Peter J. Beek
}

Received: 10 August 2006/ Accepted: 2 February 2007/Published online: 31 March 2007

(C) Springer-Verlag 2007

\begin{abstract}
The influence of attention on the dynamical structure of postural sway was examined in 30 healthy young adults by manipulating the focus of attention. In line with the proposed direct relation between the amount of attention invested in postural control and regularity of center-of-pressure (COP) time series, we hypothesized that: (1) increasing cognitive involvement in postural control (i.e., creating an internal focus by increasing task difficulty through visual deprivation) increases COP regularity, and (2) withdrawing attention from postural control (i.e., creating an external focus by performing a cognitive dual task) decreases COP regularity. We quantified COP dynamics in terms of sample entropy (regularity), standard deviation (variability), sway-path length of the normalized posturogram (curviness), largest Lyapunov exponent (local stability), correlation dimension (dimensionality) and scaling exponent (scaling behavior). Consistent with hypothesis 1 , standing with eyes closed significantly increased COP regularity. Furthermore, variability increased and local stability decreased, implying ineffective postural control. Conversely, and in line with hypothesis 2 , performing a cognitive dual task while standing with eyes closed led to greater irregularity and smaller variability, suggesting an increase in the "efficiency, or "automaticity" of postural control'. In conclusion, these findings not only indicate that
\end{abstract}

S. F. Donker · M. Roerdink · P. J. Beek $(\bowtie)$

Faculty of Human Movement Sciences,

VU University, Research Institute MOVE,

Van der Boechorststraat 9, 1081 BT Amsterdam,

The Netherlands

e-mail: p.beek@fbw.vu.nl

S. F. Donker · A. J. Greven

Department of Otorhinolaryngology, VU University

Medical Center, Amsterdam, The Netherlands regularity of COP trajectories is positively related to the amount of attention invested in postural control, but also substantiate that in certain situations an increased internal focus may in fact be detrimental to postural control.

Keywords Postural control · Center-of-pressure · Attention $\cdot$ Regularity

\section{Introduction}

By now, it is well established that maintaining and controlling an upright posture requires a certain amount of attention (for a review see Woollacott and Shumway-Cook 2002). The relation between attentional resources and the processing of information from somatosensory, visual, and vestibular systems is readily apparent in cases of reduced or conflicting sensory information (e.g., Redfern et al. 2001, 2004; Shumway-Cook and Woollacott 2000; Teasdale and Simoneau 2001). On the one hand, the degree of attention, or cognitive involvement, required for controlling posture increases with task difficulty. This has been (indirectly) illustrated by, for example, Lajoie et al. (1993) and Vuillerme and Nougier (2004), who both found that the reaction time of a verbal response to an auditory stimulus increased with the difficulty of the postural task. On the other hand, the amount of attention required to perform a secondary suprapostural task is known to influence posture (e.g., Balasubramaniam et al. 2000; Huxhold et al. 2006; Pellecchia 2003; Riley et al. 2003). The dependency of posture on attention is even more prominent in the presence of pathology or aging, when both peripheral and central changes occur that decrease the (physical) capability needed to maintain balance during standing or walking (e.g., Brown et al. 1999; Lindenberger et al. 2000; Marchese 
et al. 2003; Rankin et al. 2000; Redfern et al. 2004). In balance-impaired elderly individuals, for example, the performance of a secondary cognitive task (i.e., dual-task paradigm) may promote postural instability and even falls (Barra et al. 2006; Brauer et al. 2001). Hence, the amount of attention required for maintaining and controlling upright posture is indicative of the degree of "automaticity", of postural control and, for that reason, has been advocated and used as an important tool in clinically oriented studies (cf., Geurts et al. 1991; Melzer et al. 2001).

Center-of-pressure (COP) fluctuations measured while standing on a force platform provide a complex output signal of the postural control system in which various pertinent cognitive, perceptual, and motor processes are reflected. Recently, COP measures pertaining to the dynamical structure of COP fluctuations have helped to understand the inherent complexity of the postural control system and its constituent processes (e.g., Baratto et al. 2002; Collins and De Luca 1993; Newell et al. 1993; Pascolo et al. 2005; Peterka 2000; Yamada 1995a; cf., Riley and Turvey 2002 for a review). Inspired by this development in modern posturography, Roerdink et al. (2006) - in a study on the functional recovery of posture in stroke patients-proposed a direct relation between the amount of attention invested in postural control and the regularity of COP fluctuations. COP trajectories were more regular (as indexed by reduced sample entropy) in stroke patients than in healthy elderly and became less regular when performing a secondary cognitive task while standing. These results were interpreted to imply that postural sway regularity is positively correlated with the degree of cognitive involvement in postural control. Interestingly, the regularity of the COP fluctuations decreased with rehabilitation, whereas postural stability (as indexed by the largest Lyapunov exponent) increased, suggesting that the required degree of cognitive involvement in postural control decreased during the course of rehabilitation. Hence, these progressively more irregular COP fluctuations (as indexed by an increase in sample entropy) may be interpreted as an increase in the efficiency or "automaticity" of postural control. This interpretation is in line with physiological studies showing that a decrease in "complexity" or "irregularity" of a physiological time-series is indicative of a decrease in healthiness or effectiveness of the physiological control system (cf., Goldberger et al. 2002), a phenomenon known as "dynamical diseases" (cf. Belair et al. 1995). In other words, increased COP regularity may be explained as an indication of an increasingly ineffective postural control strategy.

The aim of the present study was to examine the role of attention in the regulation of posture. To this end, the amount of attention invested in postural control was manipulated experimentally in a large group of young healthy adults. In particular, we increased the difficulty of the postural task through visual deprivation under the assumption that an increase in task difficulty is associated with an increase in cognitive involvement in postural control, creating a so-called internal attentional focus (cf., Andersson et al. 1998; Teasdale et al. 1993; Teasdale and Simoneau 2001). In contrast, we used a concurrent attention demanding cognitive task in order to withdraw attention from postural control, creating an external focus of attention (cf., Huxhold et al. 2006). These manipulations of attention allowed us to examine the proposed direct relation between COP regularity and the amount of attention invested in postural control (Roerdink et al. 2006). In line with this relation, we hypothesized that: (1) increasing postural task difficulty (i.e., by standing with eyes closed, creating an internal focus) increases the cognitive involvement in postural control and hence the regularity of COP fluctuations, and (2) reduced attention to postural control (i.e., by performing a cognitive dual task, creating an external focus) decreases the regularity of COP fluctuations. In addition, based on the suggestion that posture is mainly controlled in the direction of the largest postural sway (Roerdink et al. 2006) and the common finding that in healthy young adults postural sway is largest in the sagittal plane relative to the frontal plane (e.g., Gatev et al. 1999; Winter et al. 1998), we expected COP regularity to be largest in the sagittal plane.

Regularity of COP trajectories was quantified by the sample entropy (Richman and Moorman 2000; Roerdink et al. 2006). In order to examine the structure of COP fluctuations in more detail, we further used a combination of more traditional (i.e., based on summary statistics) and dynamical measures that are all defined operationally in terms of readily interpretable features of motor control (see also Table 1): standard deviation of COP time-series (indexing variability or the amount of postural sway), sway-path length of the normalized posturogram (indexing the amount of twisting and turning of the COP trajectory), largest Lyapunov exponent (Rosenstein et al. 1993; indexing local stability), correlation dimension (Grassberger and Procaccia 1983; indexing the number of active, dynamical degrees of freedom involved in postural control and hence its dimensionality, e.g., Kay 1988) and the scaling exponent (e.g., Peng et al. 1995; indexing longrange correlations in COP time-series). Based on previous research, we expected visual deprivation to decrease local stability and to increase variability of COP time-series (cf., Roerdink et al. 2006; Woollacott and Shumway-Cook 2002; Yardley et al. 1999a). Moreover, we anticipated the attention manipulations to induce adjustments in the dynamical structure of postural control leading to changes in scaling exponent, sway-path length and dimensionality of COP fluctuations. 
Table 1 Abbreviations and meaning of interest of the calculated COP measures

\begin{tabular}{ll}
\hline Variable & Meaning of interest \\
\hline Sample entropy, SEn & Negatively related with the regularity of COP trajectory \\
Standard deviation, $\sigma(\mathrm{mm})$ & Positively related with the variability of COP trajectory \\
Sway-path length, $\mathrm{SP}_{n}\left(\mathrm{~s}^{-1}\right)$ & Positively related with the curviness of COP trajectory \\
Largest Lyapunov exponent, $\lambda_{\max }$ & Negatively related with the local stability of COP trajectory \\
Dimensionality, $D_{2}$ & Positively related with the number of active control variables \\
Scaling exponent, $\alpha$ & Long-range correlations: \\
& $\alpha=0.5$ for uncorrelated data (i.e., white noise), \\
& $\alpha=1.5$ for "Brown noise", the integration of white noise \\
\hline
\end{tabular}

\section{Methods}

Participants and procedures

A total of 30 healthy young adults (10 males, 20 females; mean age $=24$ years, range $=19-30$ years), without known motor impairments or movement-related disorders, volunteered to participate in the experiment. Participants stood barefoot on a $1 \times 1 \mathrm{~m}$ custom-made strain gauge force plate ${ }^{1}$ with their arms hanging relaxed alongside their body. The medial sides of the heels were separated by about $8 \mathrm{~cm}$ and each foot was placed with the toes outward at a $10^{\circ}$ angle from the sagittal midline (i.e., standard Romberg position). In order to examine the role of attention in the regulation of posture we carried out two manipulations. On the one hand, we increased the postural task difficulty by inviting participants to stand with eyes closed, while on the other hand, we withdrew attention from postural control by inviting them to perform a cognitive dual task. In particular, the participants were invited to stand upright with (1) eyes open (EO-ST), (2) eyes closed (EC-ST), (3) eyes open while performing a dual task (EO-DT) and (4) eyes closed while performing a dual task (EC-DT). The dual task consisted of uttering backwards names read out aloud by the investigator (e.g., "Simon" had to be repeated as "nomis"). The sole aim of this cognitive dual task was to withdraw attention from the postural task. Therefore, the participants were instructed to perform the task to the best of their ability. No feedback on the accuracy with which they performed this task was

\footnotetext{
${ }^{1}$ The measuring range of the amplifier was $-10 \mathrm{~V}$ to $+10 \mathrm{~V}$, and the signals from the amplifiers were digitized into a 12-bit signal by an AD converter (NI PCi 60405, National Instruments, Austin, TX, USA). The resolution was $0.28 \mathrm{~N} / \mathrm{bit}$. Calibration tests performed on the custom-made force plate showed a maximal systematic error of $3 \mathrm{~mm}$ along both $x$ and $y$ axis and a resonance frequency along the $z$ axis of $30 \mathrm{~Hz}$. In addition, experimental noise introduced variations that were less than $0.08 \mathrm{~kg}$ in magnitude in the measured test mass (i.e., $25 \mathrm{~kg}$ recorded for $5 \mathrm{~s}$ on eight different days). The random error was smaller than $0.3 \mathrm{~mm}$ along both $x$ and $y$ axis, as determined by calculating and averaging the standard deviation of all recordings of each participant after high-pass filtering (cut-off frequency of $12.5 \mathrm{~Hz})$.
}

provided. Each participant performed the four task conditions in random order and once in reverse order, resulting in a total of eight recordings. COP trajectories were collected for $35 \mathrm{~s}$ at a sampling rate of $100 \mathrm{~Hz}$.

After the local ethics committee had approved the study, all participants gave their informed consent prior to their participation.

\section{Data analysis}

In order to examine whether posture is actively controlled in the direction of largest postural sway (Roerdink et al. 2006), we analyzed both the registered $x$ (mediolateral ML) and $y$ (anterioposterior AP) COP time-series. After omitting the first $5 \mathrm{~s}$ of each recording, leaving $30 \mathrm{~s}$ of data for further analyses (i.e., 3,000 samples), the time-series were bidirectionally filtered (second-order low-pass Butterworth filter, cut-off frequency of $12.5 \mathrm{~Hz}$ ) to eliminate low amplitude measurement noise. ${ }^{2}$ First, we calculated the conventional standard deviation $\sigma$ of $x$ and $y$ COP trajectories to quantify the variability, or amount, of postural sway. Subsequently, we normalized the $x$ and $y$ trajectories to unit variance (i.e., by dividing the time-series in question by their respective standard deviation) and calculated the sway path defined as the length of the COP trajectory traveled per second. The applied normalization procedure enabled us to determine the sway path in the normalized posturogram, abbreviated as $\mathrm{SP}_{n}$, providing a scale-independent measure of the amount of twisting and turning of the COP trajectory.

Apart from these summary statistics of postural sway (i.e., $\mathrm{SP}_{n}$ and $\sigma$ ), which by definition ignore the temporal structure of the COP time-series, we assessed COP dynamics by means of sample entropy, largest Lyapunov exponent, correlation dimension and scaling exponent, which will be briefly explained in the following (for a more

\footnotetext{
${ }^{2}$ Though filtering may affect subtle nuances of a nonlinear structure, an area of concern that is in general lacking throughout the literature, it must be emphasized that the potential effect of filtering will be limited given that $95 \%$ of the power of COP time-series is located well below $5 \mathrm{~Hz}$ (e.g., Dozza et al. 2005; Maurer and Peterka 2005; Rocchi et al. 2002).
} 
extensive description we refer to Roerdink et al. (2006) and references therein). Table 1 presents the six different measures and their interpretation.

\section{Sample entropy, SEn}

Healthy physiological systems are often characterized by an irregular and complex type of variability, whereas disease or aging is often associated with greater regularity and less complexity (cf., Goldberger 1996; Goldberger et al. 2002; Pincus et al. 1991). A method to quantify the regularity of time-series is the sample entropy analysis (Lake et al. 2002; Richman and Moorman 2000). Sample entropy indexes the regularity of a given time-series, and is used to analyze complex stochastic systems that (by definition) include both deterministic and random processes (Pincus 1991). Specifically, sample entropy calculates the probability that a sequence of data points, having repeated itself within a tolerance $r$ for a window length $M$, will also repeat itself for $M+1$ points, without allowing self-matches (see Lake et al. 2002; Richman and Moorman 2000). ${ }^{3}$ Smaller sample entropy values are associated with greater regularity. In the present study, a decrease in sample entropy (i.e., more regular sway fluctuations) was interpreted as a decrease in the effectiveness of postural control.

\section{Largest Lyapunov exponent, $\lambda_{\max }$}

The largest Lyapunov exponent ${ }^{4}$ provides a measure of the local stability of a dynamical system (e.g., Abarbanel et al. 1996), i.e., the system's sensitivity to initial conditions or its resistance to small internal perturbations, such as the natural fluctuations that occur while maintaining an upright stance. It quantifies the exponential divergence or

\footnotetext{
${ }^{3}$ We selected window length $M$ to be 3 (Pincus and Goldberger 1994). An optimal value for $r$ was calculated according to a procedure described by Lake et al. (2002). In line with, e.g., Lake et al. (2002), the time-series were first normalized to unit variance. We performed these calculations using software from PhysioNet (Goldberger et al. 2000).

${ }^{4}$ The largest Lyapunov exponent $\lambda_{\max }$ was defined as the average exponential divergence $d(t)$ at time $t$ of initially close state-space trajectories, $d(t) \propto C \mathrm{e}^{\lambda_{\max } t}$, where $C$ is a constant that normalizes the initial separation (e.g., Rosenstein et al. 1993). To calculate the largest Lyapunov exponent, the embedding dimension $m$, as determined for the calculation of $D_{2}$ (i.e., $m>2 d_{m}+1$ ) was used. Distances between neighboring trajectories in state space were calculated as a function of time, i.e., $j \times \Delta t=3 \mathrm{~s}$, and then averaged over all original pairs of nearest neighbors $i$. Finally, using a least-squares fit to the "average", line defined by $y(j)=\frac{1}{\Delta t} \frac{1}{N} \sum_{i=1}^{N} \ln d_{i}(j)$ (where $\Delta t$ is the sampling period, and $d_{i}(j)$ is the distance between the $i$-th pair of nearest neighbors after $j$ discrete time steps, i.e., $j \times \Delta t=3 \mathrm{~s}$ ), $\lambda_{\text {max }}$ was estimated from its slope after fitting a range from $j \times \Delta t=0$ to $0.75 \mathrm{~s}$ (Rosenstein et al. 1993).
}

convergence of initially nearby trajectories in state space as time progresses (e.g., Rosenstein et al. 1993). If nearby points diverge, they produce instability. The exponent $\lambda_{\max }$ indexes this instability: positive values of $\lambda_{\max }$ indicate either the presence of deterministic chaos (i.e., a form of variability that is brought about by an underlying lawful nonlinear dynamical structure) or complete randomness (i.e., noise), implying that nearby points diverge rapidly, reflecting local instability and lack of predictability. In order to distinguish a deterministic component from genuine randomness, it is necessary to validate results against surrogate data (cf., Theiler et al. 1992).

\section{Dimensionality, $\mathrm{D}_{2}$}

The dimensionality of all COP time-series was calculated using the correlation dimension algorithm of Grassberger and Procaccia (1983). ${ }^{5}$ The correlation dimension provides an index of the number of independent degrees of freedom (equations of motion) that are required to reproduce the time evolutionary properties of the COP time-series. Note that this analysis of the dynamical degrees of freedom is different from the analysis of the (mechanical) degrees of freedom of the joints as commonly applied in the study of motor control, and that no straightforward or uniform relation exists between the number of component degrees of freedom in motion and the dimension of the organizational dynamic in controlling those components (Newell and Vaillancourt 2001).

\section{Scaling exponent, $\alpha$}

In order to determine whether the measured COP time-series were characterized by the presence of long-range correlations, we applied a fractal analysis method for biological time-series called detrended fluctuation (DFA) analysis (Peng et al. 1995). ${ }^{6}$ The scaling exponent $\alpha$ as determined by

\footnotetext{
${ }^{5}$ Note that after plotting the modified correlation sum against $r$ (i.e., a distance on a $\log$ scale) on a logarithmic scale, its linear slopes $d_{m}$ were estimated over a certain interval covering the most linear segments of the logarithmic plot of the modified correlation sum (i.e., between the distance $r$ capturing $0.5 \%$ of the pairs of points and the distance $r$ capturing $75 \%$ of the pairs of points). Moreover, the dimension $D_{2}$ was estimated when the slopes $d_{m}$ saturated with increasing embedding dimension $m$, satisfying the condition $m>2 d_{m}+1$. If this condition was fulfilled, then $D_{2}$ was considered a reliable estimate for a given embedding dimension. See also Roerdink et al. (2006).

${ }^{6}$ For the calculation of the scaling exponent $\alpha$ we followed the procedure described in Roerdink et al. (2006) without transforming the calculated $\alpha$ values to Hurst exponents $\mathrm{H}_{\text {DFA }}$. Note that in Roerdink et al. (2006) this transformation was based on an incorrect transformation rule. Fortunately, however, as the applied transformation was linear, it only affected the mean values of the reported scaling exponents and not the statistical results over experimental conditions.
} 
this method indicates the presence or absence of (longrange) correlations in the COP trajectories, as defined by Peng et al. (1995). For uncorrelated data, (e.g. white noise) $\alpha$ $=0.5$. An $\alpha$ greater than 0.5 and less than or equal to 1.0 indicates persistent long-range power-law correlations. In contrast, $0<\alpha<0.5$ indicates a different type of power-law correlation such that large and small values of the time series are likely to alternate. For $\alpha>1$ correlations exist but cease to be of a power-law form; $\alpha=1.5$ indicates Brown noise, i.e., integrated white noise. The scaling exponent $\alpha$ can also be viewed as an indicator that describes the roughness of the time series: the larger the value of $\alpha$, the "smoother" the time series (Peng et al. 1995).

\section{Surrogate analysis}

To test for spurious effects and to distinguish between deterministic features and randomness, the scaling exponents, dimension estimates, Lyapunov exponents and entropy values were also computed for surrogate data (Theiler et al. 1992). In particular, we generated both time- and phase-randomized surrogate data of the filtered COP timeseries (Fig. 1). In time-randomized surrogate data, the distribution of the original data is being preserved (i.e., mean, variance, etc. are unaltered), whereas the temporal correlations in the COP time-series are destroyed. The absence of temporal correlations will result in a scaling exponent $\alpha$ close to 0.5 and very large values for dimension and sample entropy. Phase-randomized surrogate data are obtained by randomizing the data's Fourier phases. In contrast to timerandomization, this procedure does not alter the spectral power distribution and preserves the data's auto-correlation function. Consequently, scaling exponents of phase-randomized and original data should match, whereas estimates of correlation dimension and sample entropy should be largely increased in the surrogate data.

\section{Statistical analysis}

For all dependent variables, the first and second trials were averaged. To test for differences between AP and ML COP measures and to examine the effect of the different experimental tasks, we used a repeated measures analysis of variance (ANOVA) design with within-subject factors vision (2 levels: standing with eyes open EO, and standing with eyes closed EC), dual task (2 levels: standing without cognitive dual task performance, i.e., single task, ST, and standing with cognitive dual task performance, DT), and plane ( 2 levels: sagittal and frontal plane). ${ }^{7}$ Subsequently,

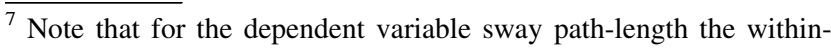
subject factor plane was redundant and, accordingly, left out of the design.
}
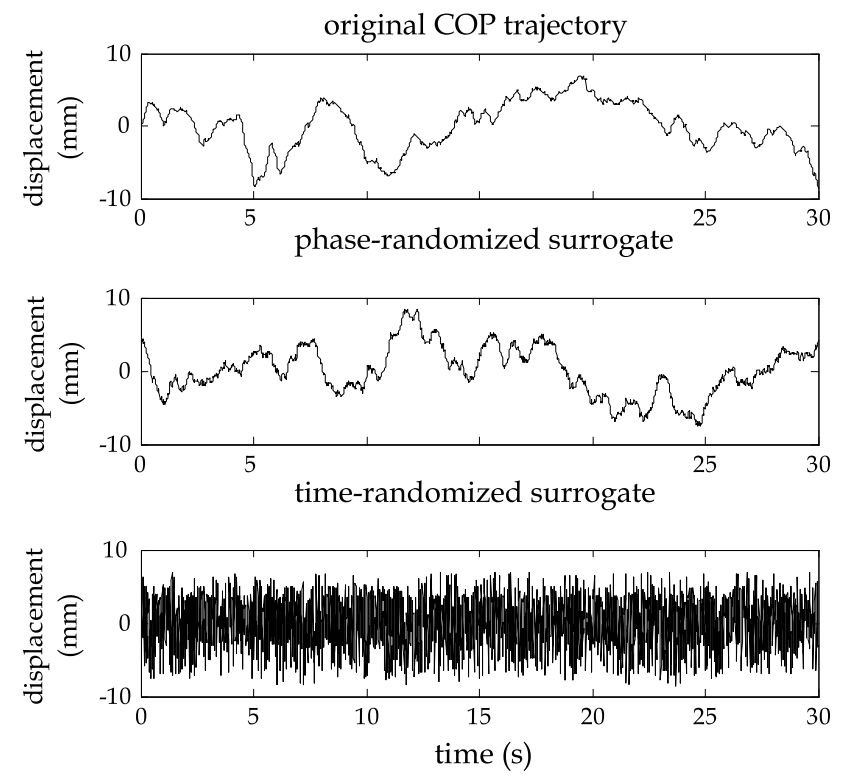

Fig. 1 An example of the surrogate analysis, as applied to all timeseries. Surrogate data of a measured COP trajectory (upper panel) were constructed by randomizing the Fourier phase (middle panel) and the temporal order (lower panel)

we tested for differences between surrogate and original data using a design involving a within-subject factor surrogate (3 levels: original data and time- and phase-randomized surrogate data). To assess the strength of the (main and interaction) effects, we determined the eta squared $\left(\eta^{2}\right)$, a commonly used measure of effect size in AVOVAs, reflecting the proportion of variance in the dependent variable that is attributable to each effect. Subsequently, $\eta^{2}$ was converted into Cohen's $f$ according to: $f=\sqrt{\frac{\eta^{2}}{1-\eta^{2}}}$. An effect size $(f)$ of $>0.4$ was considered to reflect a strong effect (Cohen 1988). The analyses were performed using SPSS (SPSS, Inc., Chicago, IL, USA).

\section{Results}

The result section is organized as follows. We first report possible differences in the dependent variables between the eyes open and eyes closed conditions (i.e., main effect of vision, hypothesis 1). Second, we describe the effect of experimentally withdrawing attention from postural control by comparing single task and dual task conditions (i.e., main effect of dual task, hypothesis 2). Third, we report whether significant vision $\times$ dual task interaction effects were present. Finally, we present the effects of plane, which may reveal possible directional differences in control. In this context, we also report the effects of randomization on the dependent variables to ensure that the observed changes in the dynamics of COP trajectories were genuine effects. 
Table 2 presents the results of the vision $\times$ dual task ANOVA for the six dependent posturographic measures (i.e., interindividual means corresponding to the main effects of vision and dual task, collapsed over $x$ and $y$ timeseries, as well as $F, P$ and $f$ values for main and interaction effects). Significant vision $\times$ dual task interaction effects are presented in Fig. 2.

Increased postural task difficulty (EO vs. EC)

As is apparent from the significant main effects of vision in Table 2, standing with eyes closed resulted in more regular sway fluctuations, as indexed by a decrease in SEn. In addition, sway variability $(\sigma)$, dimensionality $\left(D_{2}\right)$ and sway-path length $\left(\mathrm{SP}_{n}\right)$ were increased, whereas $\alpha$ and local stability (as indexed by an increase in $\lambda_{\max }$ ) decreased.

\section{Decreased attention to posture (ST vs. DT)}

Collapsed over $x$ and $y$ time-series and visual conditions, no significant main effects involving dual task were found for $\sigma$, SEn or local stability. In contrast, a main effect of dual task was found for both $\mathrm{SP}_{n}$ and $D_{2}$ in that performing a cognitive dual task brought about an increase in both variables as compared to the single task condition (Table 2). Moreover, as shown in Table 2, performing a dual task resulted in a significant decrease of $\alpha$ indicating that dual task performance brought about changes in the timevarying structure of sway fluctuations.
Vision $\times$ dual task interaction effects

Significant dual task $\times$ vision interaction effects (Table 2) revealed that the effects of visual deprivation on $S E n, \sigma$ and $\mathrm{SP}_{n}$ were different for single and dual task conditions (see Fig. 2). In particular, the observed effect of visual deprivation on $\sigma$ and SEn was significant only for the single task condition, whereas the observed increase in $\mathrm{SP}_{n}$ only existed for dual task performance (see Fig. 2). On the other hand, as can be appreciated from both Table 2 and Fig. 2, the effects of introducing a cognitive dual task on SEn, $\sigma$ and $\mathrm{SP}_{n}$ depended on eye closure. Although no significant main effect of dual task was found for SEn, there was a significant dual task $\times$ vision interaction. This effect occurred because SEn increased significantly from 0.68 to 0.72 through the introduction of a dual task when standing with eyes closed, whereas such an increase was absent when standing with eyes open ( 0.72 for both single and dual task conditions). Similarly, when standing with eyes open, dual task performance had no significant effect on $\sigma$, whereas dual task performance resulted in a decrease in $\sigma$ when standing with eyes closed (see Fig. 2).

The observed significant dual task $\times$ vision interaction effects for $\lambda_{\max }$ were brought about by the fact that the increase (i.e., decrease in local stability) when standing with eyes closed was stronger for the single task condition than for the dual task condition. A similar asymmetric effect was found for $D_{2}$, in that the increase when standing with eyes closed was stronger for the dual task condition
Table 2 Main and interaction effects of vision and dual task (i.e., collapsed over $x$ and $y$ time-series) of sample entropy (SEn), standard deviation $(\sigma)$, sway-path length of the normalized (by the standard deviation) posturogram $\left(\mathrm{SP}_{n}\right)$, largest Lyapunov exponent $\left(\lambda_{\max }\right)$ and scaling exponent $(\alpha)$ of COP time-series for 30 healthy individuals

\begin{tabular}{|c|c|c|c|c|c|c|c|c|c|c|c|c|c|}
\hline & \multirow[t]{2}{*}{ Condition } & \multirow[t]{2}{*}{ Mean } & \multicolumn{3}{|c|}{ Vision (EO vs. EC) } & \multirow[t]{2}{*}{ Condition } & \multirow[t]{2}{*}{ Mean } & \multicolumn{3}{|c|}{ Dual task (ST vs. DT) } & \multicolumn{3}{|c|}{ Vision $\times$ dual task $\mathrm{k}^{\mathrm{a}}$} \\
\hline & & & $F(1,29)$ & $P$ & $f$ & & & $F(1,29)$ & $P$ & $f$ & $F(1,29)$ & $P$ & $f$ \\
\hline \multirow[t]{2}{*}{ SEn } & $\mathrm{EO}$ & 0.72 & 3.83 & $=0.060^{*}$ & 0.36 & ST & 0.70 & 1.45 & ns & 0.25 & 6.72 & $<0.05$ & 0.48 \\
\hline & $\mathrm{EC}$ & 0.70 & & & & DT & 0.72 & & & & & & \\
\hline \multirow[t]{2}{*}{$\sigma$} & $\mathrm{EO}$ & 3.52 & 11.82 & $<0.005$ & 0.64 & ST & 3.89 & 2.45 & ns & 0.29 & 3.18 & $=0.085$ & 0.33 \\
\hline & $\mathrm{EC}$ & 4.01 & & & & DT & 3.64 & & & & & & \\
\hline \multirow[t]{2}{*}{$\mathrm{SP}_{n}$} & $\mathrm{EO}$ & 4.27 & 5.28 & $<0.05$ & 0.43 & ST & 4.13 & 13.57 & $<0.005$ & 0.68 & 6.98 & $<0.05$ & 0.49 \\
\hline & $\mathrm{EC}$ & 4.52 & & & & DT & 4.66 & & & & & & \\
\hline \multirow[t]{2}{*}{$\lambda_{\max }$} & $\mathrm{EO}$ & 1.56 & 36.23 & $<0.001$ & 1.12 & ST & 1.71 & 0.10 & ns & 0.06 & 4.26 & $<0.05$ & 0.38 \\
\hline & $\mathrm{EC}$ & 1.88 & & & & DT & 1.73 & & & & & & \\
\hline \multirow[t]{2}{*}{$D_{2}$} & $\mathrm{EO}$ & 2.23 & 23.58 & $<0.001$ & 0.90 & ST & 2.20 & 45.70 & $<0.001$ & 1.26 & 6.15 & $<0.05$ & 0.46 \\
\hline & $\mathrm{EC}$ & 2.48 & & & & DT & 2.51 & & & & & & \\
\hline \multirow[t]{2}{*}{$\alpha$} & $\mathrm{EO}$ & 1.39 & 13.70 & $<0.001$ & 0.69 & $\mathrm{ST}$ & 1.39 & 24.57 & $<0.001$ & 0.92 & 1.80 & ns & 0.25 \\
\hline & $\mathrm{EC}$ & 1.34 & & & & DT & 1.35 & & & & & & \\
\hline
\end{tabular}

* Significant vision $\times$ plane interaction $(F(1,29)=5.48, P<0.05, f=0.44)$, which was caused by the fact that, in contrast to that in the frontal plane, the effect of vision was significant in the sagittal plane $(F(1,29)=6.47, P<0.05, f=0.47)$

a See Fig. 2 for mean values of the conditions EO-ST, EC-ST, EO-DT and EC-DT 

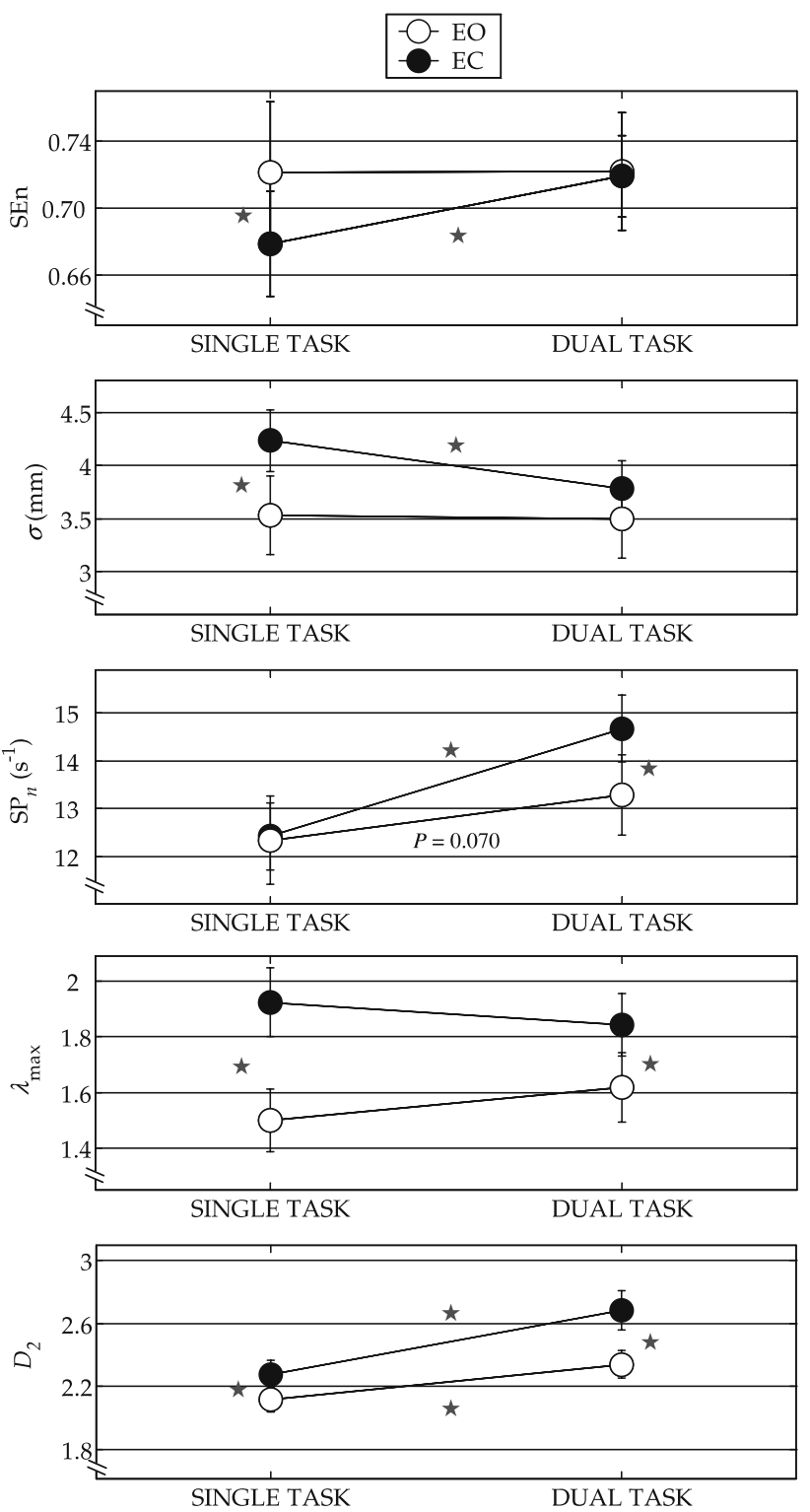

Fig. 2 Interindividual averages, collapsed over $x$ and $y$ time-series, of sample entropy (SEn), standard deviation $(\sigma)$, sway-path length of the normalized (by the standard deviation) posturogram $\left(\mathrm{SP}_{n}\right)$, local stability $\left(\lambda_{\max }\right)$ and dimensionality $\left(D_{2}\right)$ for the four experimental conditions: standing with eyes open (EO-ST), eyes closed (EC-ST), eyes open while performing a cognitive dual task (EO-DT) and eyes closed while performing a cognitive dual task (EC-DT). The asterisks indicate significant $(P<0.05)$ differences between conditions

than for the single task condition. Moreover, as can be observed in Fig. 2, the effect of performing a dual task (i.e., increase in $D_{2}$ ) was larger when standing with eyes closed than with eyes open.

Effects of plane and randomization

Collapsed over conditions, significant differences between sagittal and frontal plane were found for all variables, except $\alpha$. In particular, sway variability, $\sigma$, and local stability, $\lambda_{\max }$, were significantly larger in the sagittal than in the frontal plane $(F(1,29)=20.94, P<0.001, f=0.82$ and $F(1,29)=15.19, P<0.005, f=0.72$, respectively). In contrast, sample entropy, SEn, and dimensionality, $D_{2}$, were significantly lower in the sagittal than in the frontal plane $(F(1,29)=145.38, P<0.001, f=2.31$ and $F(1$, $29)=16.01, P<0.001, f=0.74$, respectively). A significant vision $\times$ plane interaction was found for SEn $(F(1$, $29)=5.48, P<0.05, f=0.44)$, which was caused by the fact that SEn was smaller when standing with eyes closed (EC-ST, see Fig. 2) for COP fluctuations in the sagittal plane, whereas this was not the case for COP fluctuations in the frontal plane.

Figure 3 shows the results of the interindividual means of the surrogate analyses. Sample entropy measures of both phase- and time-randomized surrogate data were significantly higher compared to the original COP time-series $(F(2,58)=10985.1, P<0.001, f=22.34)$. Whereas scaling exponents of phase-randomized surrogate data and original COP time-series did not differ, correlations were completely absent when the data were time-randomized as evidenced by $\alpha$ values around $0.5(F(2,58)=5436.7$, $P<0.001, f=14.12)$. As a result of very high-dimensional noise in the time-randomized data, no embedding dimension could be estimated and hence no dimensionality estimates and Lyapunov exponents could be determined for the time-randomized surrogate data. ${ }^{8}$ Randomizing the phases of original COP data significantly increased the dimensionality $(F(1,29)=50.07, P<0.001, f=1.31)$. In addition, the Lyapunov exponent of the phase-randomized surrogate data was significantly higher than that of the original time-series $(F(1,29)=53.44, P<0.001, f=1.36)$.

\section{Discussion}

The present experiment was conducted to investigate the role of attention in the regulation of posture. Specifically, we examined whether an increase in postural sway regularity (i.e., as indexed by a decrease in SEn) is representative of an increase in cognitive investment in postural control. We hypothesized that COP trajectories become more regular (i.e., SEn decreases) when task difficulty is increased (EC vs. EO) and, conversely, become less regular (i.e., SEn increases) when an attention-demanding cognitive dual task is introduced (DT vs. ST). We further expected that these changes in regularity of COP fluctuations would be accompanied by changes in variability, local

\footnotetext{
8 To determine the largest Lyapunov exponent, the embedding dimension $m$, as determined for the calculation of $D_{2}$ was required. However, the constraint $m>2 d_{m}+1$ was never met in the timerandomized surrogate data due to the high-dimensional noise.
} 

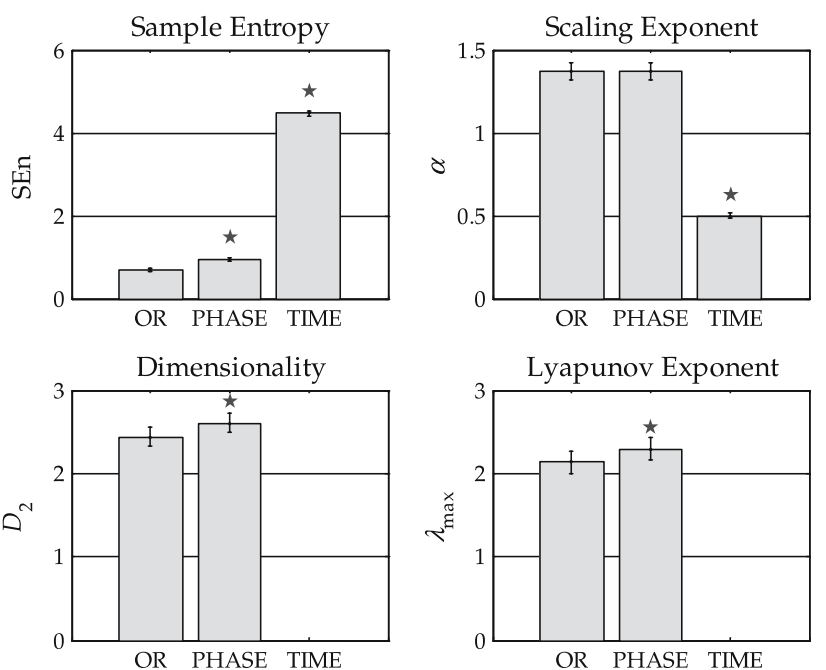

Fig. 3 Grand means, collapsed over all conditions, planes and participants, of sample entropy, scaling exponent, dimensionality and local stability for the original (OR) COP time-series and their phase-randomized (PHASE) and time-randomized (TIME) surrogate counterparts. The error bars represent the interindividual standard deviations. The asterisks represent significant $(P<0.05)$ differences between the surrogate data and the original time-series

stability, sway-path length, dimensionality and scaling exponent reflecting functional modifications of postural control. For the proper interpretation of the present findings, however, it was necessary to ascertain that the observed structure (and changes herein) of the COP fluctuations did not result from noise, but was indeed brought about by deterministic processes. Therefore, we will first discuss the results of the surrogate analyses before discussing the respective effects of vision, dual task and plane on the dynamical structure of postural sway.

\section{Surrogate analyses}

Although nonlinear estimates of dynamical structure are not readily interpretable in an absolute sense, they can be meaningfully interpreted by comparing them across conditions, (see e.g., Newell et al. 1993), as well as with surrogate data. For example, entirely random data are characterized by large (theoretically infinite) dimensionality and large $\lambda_{\max }$ values, whereas chaotic/deterministic data have smaller dimensionality and smaller $\lambda_{\max }$ values. In the present analysis, the surrogate data had greater dimensionality and larger $\lambda_{\max }$ values than the original data, implying that the latter had considerable deterministic structure (cf., Theiler et al. 1992). Moreover, both phaseand time-randomized surrogate data showed increased sample entropy values. Hence, the original COP fluctuations clearly had a deterministic component, which was evidenced further by the fact that the scaling exponents became 0.5 after time-randomization (resulting from a loss of temporal correlations in the shuffled time-series), but remained unaffected by phase-randomization (i.e., preserving temporal correlations). These findings are consistent with those of previous studies suggesting that COP fluctuations are (largely) of deterministic origin (e.g., Doyle et al. 2004; Riley et al. 1999; Yamada 1995b), and testify to the relevance and need of including dynamical measures in posturography.

Increasing postural task difficulty (EO vs. EC)

In the present study, standing with eyes closed brought about an increase of sway variability, which was accompanied by an increase in dimensionality and $\lambda_{\max }$, implying that local stability decreased. Possibly, the observed increase in dimensionality may serve as a mechanism to enrich information so as to facilitate the control of standing and to cope with the reduced (local) stability (cf., Riley and Clark 2003; van Emmerik and van Wegen 2002). These observations are in line with the common notion that visual deprivation increases the task difficulty of postural control, and, consequently, requires cognitive monitoring of postural control. We expected that this increase in cognitive investment would be accompanied by a decrease in SEn (i.e., an increase in regularity), which was indeed the case (see Fig. 2, compare EO-ST with EC-ST), thus confirming hypothesis 1 . These findings are consistent with the results of other studies showing that COP variability tends to increase as experimental task conditions become increasingly difficult, whereas the temporal structure of postural sway tends to become increasingly regular (Riley and Clark 2003; Roerdink et al. 2006; Thurner et al. 2002).

In the present study, visual deprivation resulted in qualitatively similar changes in the structure of COP trajectories as reported in the study of Roerdink et al. (2006) for stroke patients (i.e., increased $\sigma, \lambda_{\max }, D_{2}$, and decreased SEn and $\alpha$ with respect to healthy elderly adults). In healthy young adults, the qualitatively similar changes in COP dynamics with visual deprivation as compared to standing with eyes open may, likewise, be interpreted to indicate that postural control is performed less automatically and effectively. Whereas in stroke patients such modifications in postural control may be due to a defect or slowing down of the central processing of sensory information (cf., Teasdale et al. 1991; Woollacott et al. 1986), in young healthy adults a more regular sway, resulting from standing with eyes closed, implies increased "active", monitoring of postural control with increasing task difficulty (cf., Andersson et al. 1998; Nashner and McCollum 1985; Redfern et al. 2001; Teasdale et al. 1993; Teasdale and Simoneau 2001). If the proposed relation between cognitive investment in postural control and postural sway regularity does indeed exist, then the performance of a 
cognitive dual task should result in less regular COP trajectories. That this was indeed the case will be discussed in the next two subsections.

\section{Withdrawing attention from posture}

The performance of a concurrent dual task led to changes in scaling exponents and an increase in dimensionality, reflecting cognition-invoked adjustments of postural control. These changes under dual task performance may have served to enrich the information captured in sway fluctuations without increasing the amount of sway (i.e., variability remained unaltered). This interpretation is amplified by the observation that the sway-path length of the normalized posturogram increased, indicating more twisting and turning in the COP trajectories. Interestingly, despite the fact that attention was withdrawn experimentally from postural control, local stability remained unaltered. In contrast to what we expected, no main effect of dual task was found for SEn. However, this finding does not necessarily militate against the proposed relation between the regularity of COP fluctuations and the amount of attention directed to postural control, as will be argued in the following subsection.

\section{Vision $\times$ dual task interaction}

Especially noteworthy in this context and in view of our expectations is that while standing with eyes closed postural sway regularity decreased (i.e., sample entropy increased) when performing a cognitive dual task (compare EC-DT and EC-ST in Table 2 and Fig. 2). This finding is consistent with hypothesis 2 and supports the proposed positive correlation between COP regularity and the degree of attention involved in postural control. Apparently, the fact that regularity remained unaltered (i.e., 0.72) when performing a dual task while standing with eyes open (EODT) implies that, for young healthy adults, standing with eyes open is not very attention demanding. Conversely, during the more challenging task of standing with eyes closed (EC-ST) COP fluctuations became more regular (i.e., 0.68; Fig. 2). However, the finding that sample entropy again increased to its "normal" (EO-ST) level when withdrawing the focus of attention from the postural task (EC-DT) indicates that the increased cognitive monitoring of posture during EC-ST had a detrimental effect. Visual deprivation increased the awareness of the postural task (i.e., creating an internal focus), resulting in efforts to actively (consciously) control posture and, as such, preventing the postural control system to work in a relatively automatic and efficient manner (viz., Andersson et al. 2002; Hunter and Hoffman 2001; McNevin and Wulf 2002; Milton et al. 2004).
Similarly, when standing with eyes open the attentiondemanding dual task had no significant effect on $\sigma$, whereas when standing with eyes closed the dual task did result in a decrease in $\sigma$ (see Fig. 2), corroborating the findings of e.g., Andersson et al. (2002), McNevin and Wulf (2002) and Morioka et al. (2005). In contrast, many authors have found an increase in postural sway variability when performing a cognitive dual task (see Shumway-Cook and Woollacott 2000). It has been suggested that this effect of dual task on the amount of sway may, in part, result from articulation (Dault et al. 2003; Yardley et al. 1999b). In this context, it is important to note that the present finding that variability actually decreased with the introduction of a cognitive dual task indicates that articulation played no significant role in the present study.

\section{Sagittal versus frontal plane}

The increased variability of the COP trajectories in the sagittal plane (as compared to the frontal plane) was accompanied by reduced local stability and greater regularity (i.e., a decrease in sample entropy). These observations are consistent with the findings of Roerdink et al. (2006), which showed that in healthy elderly adults local stability was reduced in the sagittal plane, whereas regularity and variability were elevated in this plane. Based on these results, they suggested that posture is mainly controlled in the direction of largest postural sway (i.e., sagittal plane), which required a certain amount of attention as reflected by the regularity findings. The present observation that during standing with eyes closed (EC-ST) regularity increased significantly in the sagittal plane, whereas no effect was found for the frontal plane, is in line with this suggestion: young healthy adults mainly control posture in the sagittal plane, which becomes particularly attention demanding when task difficulty is increased (see also Pellecchia 2003; Riley et al. 2003).

\section{Conclusion}

The present study showed that the amount of attention invested in postural control is positively correlated with sway regularity. Specifically, the present study showed that increasing postural task difficulty by means of visual deprivation (EC-ST) not only resulted in an increase of COP variability and a decrease in local stability, but also in more regular COP trajectories. These findings could be taken to imply that the participants actively monitored their posture in order to cope with the increased postural task difficulty. However, when the amount of attention invested in postural control was experimentally reduced by intro- 
ducing a cognitive dual task (EC-DT), both regularity and variability of sway fluctuations returned to values observed when standing with eyes open (EO-ST). This finding suggests that during standing with eyes closed (EC-ST) the increase in monitoring posture was due to an increase in awareness of the postural task (i.e., internal attentional focus), preventing postural control from working in an automatic and efficient manner (e.g., Hunter and Hoffman 2001). All in all, it is fair to conclude that the methodological and analytical approach adopted in the present study allows for disentangling whether or not there is an increase in cognitive involvement and to what extent an increase in cognitive involvement has a detrimental or beneficial effect.

Acknowledgments This research was conducted while the second author was working on a grant of the Netherlands Organisation for Health Research and Development (ZonMw grant 1435.0004).

\section{References}

Abarbanel HD, Gills Z, Liu C, Roy R (1996) Nonlinear-time-series analysis of chaotic laser dynamics. Phys Rev A 53:440-453

Andersson G, Yardley L, Luxon L (1998) A dual-task study of interference between mental activity and control of balance. Am J Otol 19:632-637

Andersson G, Hagman J, Talianzadeh R, Svedberg A, Larsen H (2002) Effect of cognitive load on postural control. Brain Res Bull 58:135-139

Balasubramaniam R, Riley MA, Turvey MT (2000) Specificity of postural sway to the demands of a precision task. Gait Posture $11: 12-24$

Baratto L, Morasso P, Re C, Spada G (2002) A new look at posturographic analysis in the clinical context: sway-density vs. other parameterization techniques. Motor Control 6:246270

Barra J, Bray A, Sahni V, Golding JF, Gresty MA (2006) Increasing cognitive load with increasing balance challenge: recipe for catastrophe. Exp Brain Res 174:734-745

Belair J, Glass L, An Der Heiden U, Milton J (1995) Dynamical disease: identification, temporal aspects and treatment strategies of human illness. Chaos 5:1-7

Brauer SG, Woollacott M, Shumway-Cook A (2001) The interacting effects of cognitive demand and recovery of postural stability in balance-impaired elderly persons. J Gerontol A Biol Sci Med Sci 56A:M489-496

Brown LA, Shumway-Cook A, Woollacott MH (1999) Attentional demands and postural recovery: the effects of aging. J Gerontol A Biol Sci Med Sci 54:M165-171

Cohen J (1988) Statistical power analysis for the behavioral sciences. Lawrence Erlbaum Associates, Mahwah

Collins JJ, De Luca CJ (1993) Open-loop and closed-loop control of posture: a random-walk analysis of center-of-pressure trajectories. Exp Brain Res 95:308-318

Dault MC, Yardley L, Frank JS (2003) Does articulation contribute to modifications of postural control during dual-task paradigms? Brain Res Cogn Brain Res 16:434-440

Doyle TLA, Dugan EL, Humphries B, Newton RU (2004) Discriminating between elderly and young using a fractal dimension analysis of centre of pressure. Int J Med Sci 1:11-20
Dozza M, Chiari L, Horak FB (2005) Audio-biofeedback improves balance in patients with bilateral vestibular loss. Arch Phys Med Rehabil 86:1401-1403

van Emmerik RE, van Wegen EE (2002) On the functional aspects of variability in postural control. Exerc Sport Sci Rev 30:177-183

Gatev P, Thomas S, Kepple T, Hallett M (1999) Feedforward ankle strategy of balance during quiet stance in adults. J Physiol 514(Pt 3):915-928

Geurts AC, Mulder TW, Nienhuis B, Rijken RA (1991) Dual-task assessment of reorganization of postural control in persons with lower limb amputation. Arch Phys Med Rehabil 72:1059-1064

Goldberger AL (1996) Non-linear dynamics for clinicians: chaos theory, fractals, and complexity at the bedside. Lancet 347:1312-1314

Goldberger AL, Amaral LA, Glass L, Hausdorff JM, Ivanov P, Mark RG, Mietus JE, Moody GB, Peng CK, Stanley HE (2000) PhysioBank, PhysioToolkit, and PhysioNet: components of a new research resource for complex physiologic signals. Circulation 101:e215-e220

Goldberger AL, Amaral LA, Hausdorff JM, Ivanov P, Peng CK, Stanley HE (2002) Fractal dynamics in physiology: alterations with disease and aging. Proc Natl Acad Sci USA 99 (Suppl 1):2466-2472

Grassberger P, Procaccia I (1983) Characterization of strange attractors. Phys Rev Lett 50:346-349

Hunter MC, Hoffman MA (2001) Postural control: visual and cognitive manipulations. Gait Posture 13:41-48

Huxhold O, Li SC, Schmiedek F, Lindenberger U (2006) Dualtasking postural control: aging and the effects of cognitive demand in conjunction with focus of attention. Brain Res Bull 69:294-305

Kay BA (1988) The dimensionality of movement trajectories and the degrees of freedom problem: a tutorial. Hum Mov Sci 7:343-346

Lajoie Y, Teasdale N, Bard C, Fleury M (1993) Attentional demands for static and dynamic equilibrium. Exp Brain Res 97:139-144

Lake DE, Richman JS, Griffin MP, Moorman JR (2002) Sample entropy analysis of neonatal heart rate variability. Am J Physiol Regul Integr Comp Physiol 283:R789-797

Lindenberger U, Marsiske M, Baltes PB (2000) Memorizing while walking: increase in dual-task costs from young adulthood to old age. Psychol Aging 15:417-436

Marchese R, Bove M, Abbruzzese G (2003) Effect of cognitive and motor tasks on postural stability in Parkinson's disease: a posturographic study. Mov Disord 18:652-658

Maurer C, Peterka RJ (2005) A new interpretation of spontaneous sway measures based on a simple model of human postural control. J Neurophysiol 93:189-200

McNevin NH, Wulf G (2002) Attentional focus on supra-postural tasks affects postural control. Hum Mov Sci 21:187-202

Melzer I, Benjuya N, Kaplanski J (2001) Age-related changes of postural control: effect of cognitive tasks. Gerontology 47:189194

Milton JG, Small SS, Solodkin A (2004) On the road to automatic: dynamic aspects in the development of expertise. J Clin Neurophysiol 21:134-143

Morioka S, Hiyamizu M, Yagi F (2005) The effects of an attentional demand tasks on standing posture control. J Physiol Anthropol Appl Human Sci 24:215-219

Nashner LM, McCollum G (1985) The organization of human postural movements: a formal basis and experimental synthesis. Behav Brain Sci 8:135-172

Newell KM, Vaillancourt DE (2001) Dimensional change in motor learning. Hum Mov Sci 20:695-715

Newell KM, van Emmerik REA, Lee D, Sprague RL (1993) On postural stability and variability. Gait Posture 1:225-230 
Pascolo PB, Marini A, Carniel R, Barazza F (2005) Posture as a chaotic system and an application to the Parkinson's disease. Chaos Solitons Fractals 24:1343-1346

Pellecchia GL (2003) Postural sway increases with attentional demands of concurrent cognitive task. Gait Posture 18:29-34

Peng CK, Havlin S, Stanley HE, Goldberger AL (1995) Quantification of scaling exponents and crossover phenomena in nonstationary heartbeat time-series. Chaos 5:82-87

Peterka RJ (2000) Postural control model interpretation of stabilogram diffusion analysis. Biol Cybern 82:335-343

Pincus SM (1991) Approximate entropy as a measure of system complexity. Proc Natl Acad Sci USA 88:2297-2301

Pincus SM, Goldberger AL (1994) Physiological time-series analysis: what does regularity quantify? Am J Physiol 266:H1643-H1656

Pincus SM, Gladstone IM, Ehrenkranz RA (1991) A regularity statistic for medical data analysis. J Clin Monit 7:335-345

Rankin JK, Woollacott MH, Shumway-Cook A, Brown LA (2000) Cognitive influence on postural stability: a neuromuscular analysis in young and older adults. J Gerontol A Biol Sci Med Sci 55: M112-M119

Redfern MS, Jennings JR, Martin C, Furman JM (2001) Attention influences sensory integration for postural control in older adults. Gait Posture 14:211-216

Redfern MS, Talkowski ME, Jennings JR, Furman JM (2004) Cognitive influences in postural control of patients with unilateral vestibular loss. Gait Posture 19:105-114

Richman JS, Moorman JR (2000) Physiological time-series analysis using approximate entropy and sample entropy. Am J Physiol Heart Circ Physiol 278:H2039-H2049

Riley MA, Clark S (2003) Recurrence analysis of human postural sway during the sensory organization test. Neurosci Lett 342:45-48

Riley MA, Turvey MT (2002) Variability and determinism in motor behavior. J Mot Behav 34:99-125

Riley MA, Balasubramaniam R, Turvey MT (1999) Recurrence quantification analysis of postural fluctuations. Gait Posture 9:65-78

Riley MA, Baker AA, Schmit JM (2003) Inverse relation between postural variability and difficulty of a concurrent short-term memory task. Brain Res Bull 62:191-195

Rocchi L, Chiari L, Horak FB (2002) Effects of deep brain stimulation and levodopa on postural sway in Parkinson's disease. J Neurol Neurosurg Psychiatr 73:267-274

Roerdink M, De Haart M, Daffertshofer A, Donker SF, Geurts AC, Beek PJ (2006) Dynamical structure of center-of-pressure trajectories in patients recovering from stroke. Exp Brain Res 174:256-269

Rosenstein MT, Collins JJ, De Luca CJ (1993) A practical method for calculating largest Lyapunov exponents from small data sets. Phys D 65:117-134

Shumway-Cook A, Woollacott M (2000) Attentional demands and postural control: the effect of sensory context. J Gerontol A Biol Sci Med Sci 55A:M10-M16

Teasdale N, Simoneau M (2001) Attentional demands for postural control: the effects of aging and sensory reintegration. Gait Posture 14:203-210

Teasdale N, Stelmach GE, Breunig A, Meeuwsen HJ (1991) Age differences in visual sensory integration. Exp Brain Res 85:691696

Teasdale N, Bard C, Larue J, Fleury M (1993) On the cognitive penetrability of posture control. Exp Aging Res 19:1-13

Theiler J, Eubank S, Longtin A, Galdrikian B, Farmer JD (1992) Testing for nonlinearity in time series: the method of surrogate data. Phys D 58:77-94

Thurner S, Mittermaier C, Ehrenberger K (2002) Change of complexity patterns in human posture during aging. Audiol Neurootol 7:240-248

Vuillerme N, Nougier V (2004) Attentional demand for regulating postural sway: the effect of expertise in gymnastics. Brain Res Bull 63:161-165

Winter DA, Patla AE, Prince F, Ishac MG, Gielo-Perczak K (1998) Stiffness control of balance in quiet standing. J Neurophysiol 80:1211-1221

Woollacott M, Shumway-Cook A (2002) Attention and the control of posture and gait: a review of an emerging area of research. Gait Posture 16:1-14

Woollacott MH, Shumway-Cook A, Nashner LM (1986) Aging and posture control: changes in sensory organization and muscular coordination. Int J Aging Hum Dev 23:97-114

Yamada N (1995a) Chaotic swaying of the upright posture. Hum Mov Sci 14:711-726

Yamada N (1995b) Nature of variability in rhythmical movement. Hum Mov Sci 14:371-384

Yardley L, Gardner M, Lavie N, Gresty M (1999a) Attentional demands of perception of passive self-motion in darkness. Neuropsychologia 37:1293-1301

Yardley L, Gardner M, Leadbetter A, Lavie N (1999b) Effect of articulatory and mental tasks on postural control. Neuroreport $10: 215-219$ 